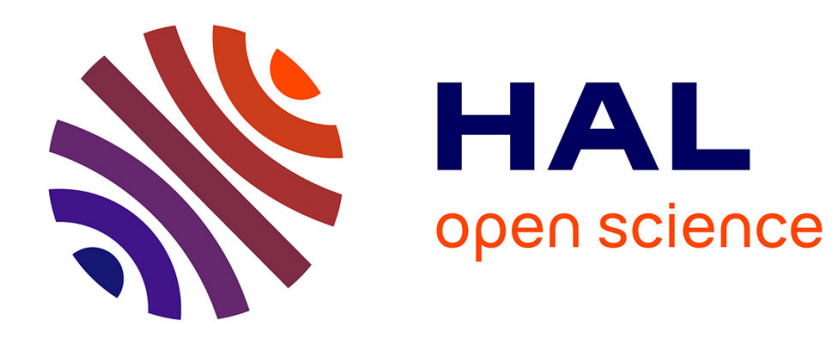

\title{
La Nature dans les écrits de Fontenelle pour l'Académie des sciences
}

Maria-Susana Seguin

\section{To cite this version:}

Maria-Susana Seguin. La Nature dans les écrits de Fontenelle pour l'Académie des sciences. DixHuitième Siècle, 2013. halshs-02329040

\section{HAL Id: halshs-02329040 \\ https://shs.hal.science/halshs-02329040}

Submitted on 23 Oct 2019

HAL is a multi-disciplinary open access archive for the deposit and dissemination of scientific research documents, whether they are published or not. The documents may come from teaching and research institutions in France or abroad, or from public or private research centers.
L'archive ouverte pluridisciplinaire HAL, est destinée au dépôt et à la diffusion de documents scientifiques de niveau recherche, publiés ou non, émanant des établissements d'enseignement et de recherche français ou étrangers, des laboratoires publics ou privés. 


\section{LA NATURE DANS LES ECRITS DE FONTENELLE POUR L'ACADEMIE DES SCIENCES.}

Lorsqu'on s'interroge sur la place et la signification que l'idée de nature peut avoir dans l'œuvre de Fontenelle, la métaphore par laquelle le philosophe des Entretiens sur la pluralité des mondes explique à la marquise sa conception mécaniste de l'univers semble s'imposer comme une évidence : «[...] je me figure toujours que la Nature est un grand spectacle qui ressemble à celui de l'opéra », affirmait le philosophe avant de développer une cosmologie mécaniste où tout dans l'univers s'explique par le jeux de poulies, de cordes et de contrepoids ${ }^{1}$. Ce passage paradigmatique de la démarche déployée par Fontenelle dans les célèbres Entretiens aura largement contribué à asseoir la réputation de l'auteur en tant que divulgateur des savoirs scientifiques et grand défenseur de la physique cartésienne, y compris à une époque où la pensée de Newton a réussi à s'imposer en France au sein même des cercles savants $^{2}$.

Et il est vrai que Fontenelle est sans doute l'un de ceux qui aura le plus contribué, à la charnière des $\mathrm{XVII}^{\mathrm{e}}$ et $\mathrm{XVIII}^{\mathrm{e}}$ siècles, à inscrire les savoirs scientifiques dans l'espace publique $^{3}$. Son travail en tant que secrétaire de l'Académie royale des sciences renouvelée ${ }^{4}$, et responsable à ce titre de la rédaction des volumes annuels de l'Histoire de l'académie des sciences $^{5}$, lui offre les moyens de diffuser les activités des membres de la savante compagnie

\footnotetext{
1 Fontenelle, Entretiens sur la pluralité des mondes (1686). Éd. critique par Christophe Martin, Paris, Flammarion, 1998, « Premier soir », p. 62.

${ }^{2}$ On peut observer à ce propos que les Entretiens sur la pluralité des mondes paraissent un an avant les Principia de Newton (1687). La publication tardive du Traité des tourbillons (1752) a longtemps servi à expliquer le « cartésianisme » de Fontenelle, mais la véritable nature de cet engagement est actuellement reconsidérée. Voir F. Pépin, «Être cartésien pour un historien de la pensée: Fontenelle, les cartésiens et la philosophie cartésienne », dans Delphine Kolesnik (dir.), Qu'est-ce qu'être cartésien?, vol. II, «Réceptions et transformations : trois siècles de cartésianismes », Lyon, ENS Éditions, à paraître en 2012.

${ }^{3}$ Nous préférons parler ici de diffusion des savoirs dans l'espace publique, et non pas de « vulgarisation », ce qui suppose que les savoir seraient déjà organisés en tant que tels, autour d'un langage qui leur serait propre, d'un système méthodologique, bénéficiant même d'une formation professionnelle spécifique, que le savant devrait «traduire » dans le langage du «vulgaire» incapable de comprendre autrement les textes scientifiques. Cette démarche ne définit pas vraiment le travail du secrétaire de l'Académie des sciences en particulier pour ce qui est des sciences de la nature et du vivant et en général des sciences «non mathématiques ».

${ }^{4}$ Fondée en 1666, l'Académie royale des sciences sera profondément renouvelée et réorganisée en 1699, sous l'impulsion de son président, l'Abbé Bignon. Le nouveau règlement prévoit, entre autres dispositions, la création du poste de secrétaire perpétuel, charge qui revient à Fontenelle, membre de l'institution depuis 1697 en qualité de géomètre. Voir Simone Mazauric, Fontenelle et l'invention de l'histoire des sciences à l'aube des Lumières, Paris, Fayard, 2007 et Maria Susana Seguin, Histoire de l'Académie des sciences (1699-1740). Étude critique, Turnhout, Brepols, coll. « Travaux de l'Académie internationale des sciences », à paraître (2013).

${ }^{5}$ Le titre exact de la publication est Histoire de l'Académie royale des sciences, année [...] avec les Mémoires de Mathématique et de Physique pour la même année, tirés des registres de cette Académie. Conformément aux
} 
auprès d'un large public européen, pour lequel il propose, chaque année, un parcours thématique et historique des principales découvertes en matière de physique, d'anatomie, de botanique ou de mathématiques. Fontenelle s'acquitte de cette tâche pesante avec une régularité exemplaire (un volume par an) jusqu'en 1740, au moment où, très âgé déjà, il sera remplacé dans ses fonctions par Dortous de Mairan. Ce contexte privilégié lui donne également l'occasion de proposer à ses lecteurs une vision très personnelle de l'histoire des savoirs en construction qui fait du secrétaire de l'institution l'un des premiers historiens et philosophes des sciences ${ }^{6}$.

En effet, l'Histoire de l'Académie royale des sciences est entièrement consacrée à présenter au public le travail acharné des savants pour déchiffrer les nombreux secrets que recèle la nature, car comme l'affirme Fontenelle dans la Préface sur l'utilité des mathématiques « la Nature n'est [...] jamais si admirable, ni si admirée que quand elle est connue $»^{7}$. Tout comme le Philosophe le faisait avec la Marquise des Entretiens, le secrétaire de la savante compagnie invite son lecteur à ne pas se contenter du simple émerveillement devant le spectacle de la nature mais plutôt à tenter de comprendre comment fonctionne «l'arrière du théâtre », ce à quoi se consacrent les différents Mémoires publiés dans le volume. Le fait est que, dans cette enquête, la nature que décrit Fontenelle n'apparaît pas seulement à travers la métaphore mécaniste, comme un objet complexe dont il convient de démonter les nombreux et obscurs mécanismes, mais comme un être agissant par lui-même, dans un processus de personnification qui, loin de constituer de simples ornements destinés à divertir ou à intéresser un lecteur mondain, finissent par susciter des interrogations à propos de l'attitude du très officiel secrétaire de l'Académie royale des sciences.

La métaphore de l'opéra utilisée par le Philosophe des Entretiens sur la pluralité des Mondes pour représenter le modèle cartésien de la nature se déploie dans le texte à travers l'allusion aux «mécaniciens » qui, cachés au milieu du public, sont capables de voir le véritable spectacle de l'univers sans céder au merveilleux de la représentation. Cette même métaphore apparaît dans les différents volumes de l'Histoire de l'Académie des sciences, où

\footnotetext{
usages, nous renverrons désormais aux différents volumes de la collection en abrégé, HARS, suivi de l'année académique (et non l'année de publication).

${ }^{6}$ F. Pépin, «Fontenelle, premier représentant d'une tradition française d'épistémologie historique », Dixhuitième siècle, $\mathrm{n}^{\circ}$ 44, 2012, p. 381-398.

${ }^{7}$ HARS pour 1699, «Préface », p. xvi. La Préface sur l'utilité des mathématiques est extraite de la préface générale de l'Histoire de l'académie des sciences que Fontenelle publie à part, avec les Éloges des Académiciens, et sous son nom propre. : Histoire du renouvellement de l'Académie royale des sciences en 1699, et les éloges historiques de tous les académiciens morts depuis ce renouvellement, avec un discours préliminaire sur l'utilité des mathématiques et de la physique, Paris : Vve J. Boudot, 1708.
} 
elle reste active dans la présence des «spectateurs », terme qui désigne régulièrement les savants, en particulier les astronomes ${ }^{8}$, mais aussi les physiciens, les anatomistes ou les biologistes, autrement dit, tous les savants qui observent avec attention une nature que la raison n'a pas encore pu complètement élucider. Sous la plume du Fontenelle académicien, le « spectateur » apparaît comme la personnification de la première attitude de l'esprit humain tentant de saisir les mécanismes de fonctionnement du monde pour en élaborer un discours de savoir. Le regard du savant suppose ainsi une attitude d'esprit totalement différente de la contemplation naïve qui fait s'émerveiller le simple observateur devant les effets naturels, comme c'était le cas du public d'opéra devant le vol de Phaéton. Non pas que la nature n'offre de nombreuses «merveilles » : le corps humain ${ }^{9}$, les insectes ${ }^{10}$, le cerveau humain ${ }^{11}$ renferment des secrets capables d'éveiller l'admiration des savants. Mais celles-ci disparaissent une fois qu'on parvient à rendre compte des mécanismes naturels qui expliquent ces effets surprenants, voire lorsqu'on peut prouver que ces cas apparemment exceptionnels répondent à une forme de constante, même quand on n'a pas encore pu expliquer le fonctionnement des lois qui les régissent ${ }^{12}$. Le rôle du savant est alors comparable à celui du «machiniste» caché dans le parterre, capable d'expliquer le fonctionnement des «machineries de l'opéra », ou des «merveilles de la Nature », de voir, comme le philosophe des Entretiens le suggère, comment «le derrière du théâtre serait disposé ${ }^{13}$.

La métaphore du spectacle ainsi utilisée renvoie chez Fontenelle au principe épistémologique de l'observation, l'un des deux piliers, avec l'expérience, sur lesquels se construit le savoir scientifique tel que le conçoit l'Académie des sciences ${ }^{14}$. Elle se diffuse

\footnotetext{
${ }^{8}$ «Il n'appartient qu'aux phénomènes célestes, entièrement dégagés de notre atmosphère et d'ailleurs très simples et très uniformes, d'être les mêmes pour tous les spectateurs », « Sur la lumière septentrionale ", HARS, 1720, p. 5.

${ }^{9}$ «Mais que sera-ce, si l'on fait réflexion, que l'homme est un assemblage de merveilles, ou semblables à cellelà, ou équivalentes, dont le nombre est beaucoup plus grand que celui des divisions du diamètre de la glotte ? », «Sur la formation de la voix », HARS 1700, p. 25.

${ }^{10}$ "Quelque ancienne et quelque établie que soit la réputation des abeilles, on le les croyait point encore aussi merveilleuses qu'elles le sont, et on peut dire d'elles ce qu'on dit quelquefois des personnes de mérite, qu'elles gagnent à être connues », « Sur les abeilles », HARS, 1712, p. 5.

${ }_{11}$ «Le plus grand appareil de chimie qui soit dans tout le corps humain, le plus merveilleux laboratoire est dans le cerveau », « Sur la glande pituitaire », HARS 1707, p. 16.

${ }^{12} \mathrm{C}$ 'est ce qui arrive quand Fontenelle commente le cas d'une petite fille qui, âgée à peine de quelques mois, aurait eu ses règles, traduisant une maturité sexuelle étonnante: "Les filles des Indes Orientales que les Voyageurs assurent qui ont des enfants à 9 ans, ne sont plus une merveille », conclue-t-il. «Diverses observations anatomiques », II, HARS 1708, p. 52.

${ }^{13}$ Entretiens, op. cit., p. 64.

${ }^{14}$ Ce sont aussi ces deux principes, hérités de la pensée baconienne, qui expliquent la division des chapitres de l'Histoire de l'Académie des sciences en deux grands ensembles, la «physique » et les «mathématique ». Voir Roger Hahn, L'Anatomie d'une institution scientifique. L'Académie des sciences de Paris, 1666-1803, Paris, Éd. des Archives contemporaines, 1993, p. 77. Voir également M. Susana Seguin, «Fontenelle et l'Histoire de l'Académie royale des sciences », Dix-huitième siècle, nº 44/2012, p. 364-379.
} 
dans un réseau sémantique qui, dans l'ensemble des chapitres de l'Histoire de l'Académie des sciences, notamment ceux consacrés à la «physique », prend la forme de l'isotopie de la vue, l'activité du savant étant assimilée à la capacité de «voir » ce que les autres ne voient pas (comme le machiniste du parterre), de percer à jour le fonctionnement de la nature, de déjouer de qu'elle a de «merveilleux » et de transcrire cette réalité dans un code langagier capable de transformer le spectacle prodigieux en connaissance, sinon certaine du moins probable, du monde naturel. Ce principe impose le recours à des observations multiples, répétées dans le temps, comme seule possibilité d'accéder à une forme de connaissance sûre :

L'histoire naturelle est immense, et ce qui en est exposé à nos yeux n'est presque rien en comparaison de ce qui est plus caché, et ne se peut découvrir qu'avec beaucoup de temps, de loisir, de patience, d'adresse et de certains yeux que tout le monde n'a pas ${ }^{15}$.

On comprend ainsi que l'observation apparaît pour Fontenelle comme un stade premier de la pensée, un outil complémentaire et indispensable de la raison humaine, notamment lorsqu'il s'agit d'appréhender les faits naturels : «Les mouvements intérieurs des plantes sont ceux qui font leur végétation; les yeux ne les aperçoivent point, et la raison a bien de la peine à en faire plus que les yeux $»^{16}$, affirme le secrétaire de l'Académie. Le philosophe des Entretiens n'expliquait pas autre chose à la marquise, quand il lui indiquait que « les vrais philosophes passent leur vie à ne point croire ce qu'ils voient, et à tâcher de deviner ce qu'ils ne voient pas $\gg{ }^{17}$... ce qui se traduit, dans les volumes de l'Histoire de l'académie des sciences par une forme d'exigence dans l'observation, dans laquelle nous reconnaissons la supériorité de la raison appliquée à l'étude de la nature : il faut observer «avec de certains yeux ${ }^{18}$.

Ainsi, voir «avec des yeux de physicien ${ }^{19}$, pour reprendre l'expression de Fontenelle, implique l'intention de dépasser les apparences, d'appréhender le monde sensible en faisant abstraction de toute détermination causale ou finale et de répondre aux interrogations suscitées par la nature par le biais d'un discours en adéquation avec le monde sensible, une attitude qui distingue le philosophe de l'homme ordinaire, le «machiniste » du

\footnotetext{
15 «Sur le mouvement progressif de quelques coquillages ou animaux de mer», HARS pour 1712, p. 12. La «vue » particulière des savants est également évoquée dans certains cas, à propos des mathématiques, lorsque les yeux deviennent une figure de la raison en action : «Ce ne sont pas de médiocres progrès en géométrie, que les découvertes de ces sortes de rapports qui s'étaient dérobés jusqu'à présent aux yeux des mathématiciens, et que notre siècle dévoile enfin à force d'art et de recherches », "Sur les tangentes et les sécantes des angles », HARS pour 1705, p. 65 .

${ }^{16}$ «Sur les mouvements extérieurs des plantes », HARS pour 1710, p. 64.

${ }^{17}$ Entretiens, op. cit., p. 62.

18 «Sur la perpendiculaire des tiges des plantes, par rapport à l'horizon», HARS pour 1700, p. 71.

19 « Observations sur les singularités de l'histoire naturelle de France », HARS pour 1699, p. 23.
} 
public d'opéra. C'est le cas de cette mystérieuse grotte, près de Grenoble ${ }^{20}$, que des récits datant du $\mathrm{XVI}^{\mathrm{e}}$ siècle décrivent comme fort mystérieuse, mais qui, après vérification, devient une «singularité » de la nature, digne de la curiosité des savants, mais rien de plus : «M. Dieulamant a pris la peine d'envoyer à l'Académie une relation de la Grotte qu'il a examinée de ses propres yeux, et elle ne conserve plus aucun vestige de ses anciennes merveilles $»^{21}$.

Les «yeux » des savants ne sont certes pas infaillibles, «les yeux aidés des meilleurs microscopes ne peuvent aller que jusqu'à un certain point, après quoi c'est à la raison à deviner, et par conséquent c'est là que commence le péril de se tromper, si cependant les yeux eux-mêmes n'ont pas déjà aussi un peu deviné à leur manière $»^{22}$, observe Fontenelle à propos d'un mémoire du médecin Winslow sur le fonctionnement des glandes. Cela dit, la méfiance à l'égard du monde sensible et le pouvoir trompeur de nos sens ne sont que relatifs. La possibilité d'un savoir sur la nature ne s'en trouve pas diminuée, si tant est que le savant devient capable d'aider sa «vision » purement sensible, soit à travers des outils techniques, soit à travers des outils conceptuels. La métaphore de la vue est utilisée pour désigner les différentes activités intellectuelles qui permettent d'élaborer un savoir sur la nature :

Il n'y a rien dans les animaux qui n'ait sa structure particulière et organique, et si le premier coup d'œil ne nous la découvre pas, la recherche de la dissection, ou le microscope, ou le raisonnement nous la découvriront : trois manières différentes de voir, qu'il faut ajouter à notre vue simple et ordinaire, et qui vont infiniment plus $\operatorname{loin}^{23}$.

Le regard sur la nature qu'élabore le discours agit donc comme un outil de l'esprit dans son effort d'interprétation : «Les observations et les faits deviennent, selon qu'on sait les mettre en œuvre, des sources plus ou moins fécondes de réflexions et de découvertes $»^{24}$. Ou encore : «Tout sujet exactement considéré devient infini, - remarque Fontenelle à propos d'un mémoire sur les cordes vocales et la formation de la voix — et l'attention est une espèce de microscope, qui le grossit et le multiplie toujours, à proportion qu'elle est plus parfaite $»^{25}$. L'élaboration d'un savoir sur la nature repose alors sur la diversification des modalités d'analyse, ainsi que sur la multiplications des observations que les savants portent

\footnotetext{
${ }^{20}$ Il s'agit de la grotte de La Balme, l'une des sept merveilles du Dauphiné. Nous retrouvons ici la même problématique que Fontenelle développe dans d'autres de ses œuvres consacrées à l'histoire de «l'esprit humain », et notamment le Traité des oracles (1687).

21 «Sur quelques singularités de la France», HARS pour 1700, p. 4.

22 «Sur les filtrations ou secrétions des sucs dans les glandes », HARS pour 1711, p. 19.

23 «Sur la structure de la moelle », HARS pour 1714, p. 14. Je souligne.

24 «Sur le rapport des mesures anciennes avec les modernes », HARS pour 1702, p. 65.

25 « Sur la formation de la voix », HARS pour 1706, p. 15.
} 
sur elle au cours de leur vie, et au delà, dans le cours de l'histoire de l'esprit humain ${ }^{26}$. Cette conception épistémologique est à la source de l'esthétique du «faire voir » que Fontenelle développe dans l'Histoire de l'académie des sciences : utilisation récurrente de l'hypotypose, multiplication des images sensorielles, précision minutieuse de la description qui ne tient compte ni de la morale ni de la bienséance ${ }^{27}$. Ce travail correspond non seulement à la perspective historique qui, seule, garantit la possibilité d'un savoir sur la nature, mais également à sa volonté de donner au discours sur la nature sensible l' « esprit géométrique ${ }^{28}$ qui garantit, aux yeux de notre auteur, la qualité du discours scientifique.

Démultiplier les regards revient à adopter une attitude épistémologique qui épouse les manifestations mêmes de la nature, comme la seule possibilité de l'appréhender dans ses multiples manifestations : «Il n’y a encore rien qui ait été assez examiné, et peut-être rien ne le sera jamais assez. Tout est infini dans la nature », observe Fontenelle au sujet d'un mémoire consacré aux yeux des hommes et des animaux ${ }^{29}$. Son travail de secrétaire de l'Académie des Sciences semble le conforter dans l'idée que l'uniformité des lois de l'univers n'entraîne pas la fixité de ses structures, et que la nature agit par des processus combinatoires rendant possibles une infinité de manifestations. L'élaboration d'un savoir sûr suppose alors au moins autant d'observations que la nature a de manifestations, ce qui n'est concevable que dans une dimension historique : «Il semble que la nature ait pris plaisir à suivre les règles des combinaisons, et plus on comparera ensemble ses différents ouvrages, plus on trouvera que ce génie des combinaisons y domine $»^{30}$, conclut Fontenelle à propos des remarques proposées par Réaumur sur les guêpes.

C'est ici que l'on peut percevoir l'audace philosophique de Fontenelle. Cette dynamique combinatoire dont il dote la nature, et qui rend nécessaire la démultiplication des regards, écarte totalement et les causes premières, et les causes finales du discours qui peut lui

\footnotetext{
${ }^{26}$ A propos de la dimension historique du savoir chez Fontenelle voir Maria Susana Seguin, « Fontenelle à 1'Académie des Sciences : de l'approche sérielle à l'approche historique », dans Séries et variations. Études littéraires offertes à Sylvain Menant, Paris, PUPS, 2010, p. 123-136.

27 « Il y a longtemps que la physique et la médecine sont dispensées des bienséances exactes du discours, et que la morale elle-même a consenti aux libertés qu'elles se donnent», affirme Fontenelle (« Sur la gonorrhée », HARS pour 1711, p. 22.). En effet, les descriptions anatomiques ne se caractérisent pas par la présence de paraphrases élégantes, d'euphémismes ou de litotes, mais plutôt par des images sensorielles nombreuses qui n'épargnent au lecteur aucune forme d'odeur ou de saveur...

${ }^{28}$ Fontenelle définit ainsi ce point central de sa pensée épistémologique : «L'esprit géométrique n'est pas si attaché à la géométrie qu'il n'en puisse être tiré, et transporté à d'autres connaissances. Un ouvrage de Morale, de Politique, de Critique, peut-être même d'Éloquence, en sera plus beau, toutes choses d'ailleurs égales, s'il est fait de main de géomètre. » HARS pour 1699, « Préface », p. xii.

${ }^{29}$ «Sur les yeux de l'homme et de différents animaux », HARS pour 1726, p. 21.

${ }^{30}$ «Sur les guêpes », HARS pour 1719, p. 20.
} 
être consacré. Et de fait, l'Histoire de l'académie des sciences s'intéresse plus au «comment» qu'au «pourquoi » des phénomènes naturels. Le «spectacle de la Nature », pour reprendre le titre de l'Abbé Pluche dont l'œuvre est contemporaine du travail de Fontenelle $^{31}$, n'invite nullement à adorer le Créateur de l'univers, dont l'existence est le plus souvent soigneusement passée sous silence.

En effet, Fontenelle évoque rarement $l^{\prime}$ «auteur de la nature ${ }^{32}$, et le plus souvent il limite son action à l'élaboration de lois générales de l'univers, «les seules dignes de sa sagesse $»^{33}$. Les expressions «Dieu », «Créateur » «Souverain Ouvrier » apparaissent très rarement dans les articles de l'Histoire de l'académie des sciences, une vingtaine d'occurrences dans l'ensemble des volumes écrits par Fontenelle entre 1699 et 1740 ... Le plus souvent, il s'agit d'évoquer l'origine des êtres vivants ou des lois générales de l'univers, autrement dit, une cause première rendant compte de l'existence de la matière et du mouvement, qui constituent les seuls éléments sur lesquels peut s'élaborer le savoir scientifique $^{34}$ : «Si tous les animaux ont été immédiatement formés par la main du Souverain Ouvrier, on ne peut guère s'empêcher de croire que tous ceux d'une même espèce ont été formés entièrement semblables », affirme-t-il dans un mémoire consacré à une malformation anatomique $^{35}$. Ou encore, à propos du mécanisme glandulaire :

Puisque selon la plus saine philosophie, il faut supposer que tous les corps organisés ont été formés immédiatement par les mains du souverain Ouvrier, longtemps avant ce qu'on appelle leur naissance, il n'y a qu'à supposer aussi que les filtres de ces machines imperceptibles ont été dès cette première formation abreuvés des liqueurs qu'ils devaient séparer. Ce n'est point là faire entrer Dieu mal à propos dans la physique, c'est ramener la physique à sa première source ${ }^{36}$.

La tonalité générale de Fontenelle dans ces passages n'a apparemment pas de quoi faire réagir les défenseurs de la religion (qui, de fait, n'ont jamais critiqué l'œuvre historique de l'institution) puisque le secrétaire de l'Académie sauvegarde l'idée d'un Créateur suprême présidant au fonctionnement de l'Univers. Mais la présence d'un verbe modalisateur (« il faut supposer que ») ou de la conjonction à valeur hypothétique («si »), et l'utilisation récurrente

\footnotetext{
${ }^{31}$ Noel-Antoine Pluche, Le Spectacle de la Nature, Paris, chez la Veuve Estienne, 1732-1750.

32 «Sur les monstres », HARS pour 1740, p. 49.

${ }^{33}$ Ibid.

${ }^{34}$ L'attitude fort complexe de Fontenelle à l'égard du Créateur de la Nature est comparable à celle qu'il développe dans De l'existence de Dieu. Voir l'édition de ce texte par Sophie Audidière dans Fontenelle, Digression sur les anciens et les modernes et autres textes philosophiques, Paris, Garnier, à paraître.

35 «Sur une matrice double », HARS pour 1705, p. 48.

${ }^{36}$ «Sur les tuyaux capillaires », HARS pour 1705, p. 25.
} 
du passé pour présenter les actions divines, restreignent sensiblement la puissance de « l'auteur de la nature ». Par ailleurs, à aucun moment Fontenelle ne fait intervenir la notion de Providence comme forme d'explication possible de certains faits naturels, encore moins l'idée d'une nature qui inviterait à adorer le Dieu créateur comme le fait à la même époque la «science théologique ${ }^{37}$. Cette forme de neutralité par rapport au discours en vigueur s'explique, certes, par le caractère officiel de l'Histoire de l'académie des sciences, mais elle ne dit pas tout, et notamment pas la place prépondérante qu'occupe dans les mêmes volumes les personnifications, très nombreuses, de la nature.

Il est en effet remarquable que, le plus souvent, la nature qui apparaît comme une forme d'intelligence supérieure, constamment personnifiée, et le plus souvent désignée par l'usage de la majuscule. Elle est ainsi dotée d'une volonté propre, qui se traduit par l'évocation de ses « desseins » particuliers :

Il ne s'agit que de deviner juste les desseins de la Nature, mais il est toujours à présumer qu'un seul moyen en exécute plusieurs ${ }^{38}$

par ses objectifs propres, ses « vues » :

[...] si la Nature peut pousser quelquefois trop loin l'ossification des chairs, qu'elle a eue souvent en vue, cette raison cesse à l'égard de la carnification des os, qui n'a jamais été de son dessein ${ }^{39}$.

Elle a même un plan général dans ses productions :

Jamais peut-être on ne prouvera mieux que par le sujet de cet article, que la Nature ayant pris un certain plan général, fait ensuite le diversifier de toutes les façons que demandent les applications particulières ${ }^{40}$.

La Nature est même dotée d'intelligence, Fontenelle parle à plusieurs reprises de la «sagesse de la Nature ${ }^{41}$, de son «génie » ${ }^{42}$, voire même d'une certaine forme de ruse, puisqu'elle se moque des savants, déjoue leurs systèmes, se dérobe aux yeux des philosophes,

\footnotetext{
${ }^{37}$ Daniel Mornet, «L'histoire naturelle fantaisiste au XVIIIe siècle », Revue du Mois, juin 1910, p. 641-657.

38 «Sur les règles des femmes », HARS pour 1720, p. 18.

39 «Sur des os devenus chair», HARS pour 1721, p. 14.

${ }^{40}$ «Sur la circulation du sang dans les poissons », HARS pour 1701, p. 46.

${ }^{41}$ «La sagesse de la nature a sacrifié un avantage qui ne nous servait de rien, à un autre qui nous est fort utile », « Sur la formation de la voix », HARS pour 1700, p. 19.

42 «[...] il serait assuré du génie de la Nature, d'avoir ménagé des ressources pour les accidents du cerveau $[\ldots] »$, « Sur un cerveau pétrifié », HARS pour 1703, p. 27.
} 
et «cache » ses secrets les plus chers, comme la nature de l'âme humaine ou celle de la matière ${ }^{43}$ :

La première formation des animaux qui sortent vivants du sein de leurs mères, semble avoir été conduite par la Nature avec plus de secret, que celle des autres animaux ${ }^{44}$.

En voici encore un [exemple] du moins aussi surprenant, et qui paraît en quelque sorte être un effort de la Nature, pour échapper à nos recherches, et pour nous cacher son secret $^{45}$.

On dirait que la nature a eu peur que cette pierre transparente ne fût pas une énigme assez inexplicable pour les physiciens, et qu'elle l'a chargée à plaisir d'obscurités, et de difficultés ${ }^{46}$

Il est vrai, comme l'a montré Jean Dagen, que la personnification de la nature est une des formes stylistiques privilégiées par Fontenelle ailleurs dans son œuvre, notamment dans les Entretiens sur la pluralité des mondes et dans les Nouveaux dialogues des morts, et qu'elle subit un traitement parodique, qui aboutit à sa « dénaturation »: Fontenelle en vient à vider dangereusement de son sens les conceptions traditionnelles de l'idée de nature, à « reléguer cette entité au nombre des figures fabuleuses de la pensée ${ }^{47}$. L'emploi qu'en fait Fontenelle dans l'Histoire de l'académie des sciences peut paraître sans doute plus subtil, même si les conséquences confirment une attitude philosophique hautement dangereuse. Si l'idée représente en même temps «l'ensemble des êtres, des substances et des phénomènes de l'univers physique ou humain, [et] l'intelligence planificatrice assignant à chaque élément, à la matière, à la vie, à l'esprit, sa composition, sa structure, ses caractères, sa finalité $»^{48}$, le caractère officiel des écrits académiques et la portée scientifique et historique de l'ensemble paraissent interdire la dimension parodique que l'on constate dans les écrits personnels de l'auteur.

\footnotetext{
${ }^{43}$ «Les premières notions de la physique, l'essence de la matière, par exemple, et la nature du mouvement, quoique les plus simples en elles-mêmes, ne sont pas les plus claires; et ces principes qu'il semblerait qu'on devrait connaître parfaitement, avant que d'aller plus loin, demeurent cependant assez peu connus, et on ne laisse pas d'avancer », «Sur la continuation du mouvement », HARS pour 1701, p. 14. «Comme des deux parties qui composent l'homme, la plus inconnue est l'âme; aussi de toutes les parties du corps, celle qui a le plus de rapport à l'âme [le cerveau], est la plus inconnue », HARS pour 1700, "Observations d'anatomie », XXI, p. 25. Ces deux notions, hautement périlleuses dans le contexte philosophique du temps, font l'objet d'un traitement complexe par Fontenelle qui mériterait une étude complémentaire. Voir à ce sujet mon article «La question de l'âme humaine dans l'Histoire de l'Académie des sciences dans la première moitié du XVIII ${ }^{\mathrm{e}}$ siècle », à paraître dans La Lettre clandestine $\mathrm{n}^{\circ}$ 18, 2009.

${ }_{44}^{44}$ Sur un embryon », HARS pour 1701, p. 19.

45 «Sur un cerveau pétrifié », HARS pour 1703, art. cité, p. 27.

${ }^{46}$ « Sur les réfractions d'une espèce de talc », HARS pour 1710, p. 123.

${ }^{47}$ Jean Dagen, «D’une nature joliment conjecturale », Corpus, revue de philosophie, n ${ }^{\circ} 44-2003$, p. 57-72, et en particulier, p. 70.

${ }^{48}$ Ibid., p. 63.
} 
En revanche, l'utilisation généralisée de cette figure, dont les exemples jalonnent la plupart des articles de physique de chaque volume, en vient à imposer l'idée d'une force agissante qui se substitue à l'idée de divinité. La nature est partout représentée comme constante et régulière dans ses lois : «La nature est aussi uniforme qu'ingénieuse, et même d'autant plus ingénieuse qu'elle est plus uniforme ${ }^{49}$, affirme par exemple Fontenelle par un formule qui relève de la pratique de la sentence. Mais sa constance ne se traduit pas par une forme de passivité, au contraire, elle est le plus souvent le sujet de verbes d'action qui nous rappellent que la Nature est constamment productrice de nouvelles formes qui complexifient constamment le travail des savants. Ainsi, « la Nature est féconde et inépuisable en inventions mécaniques, pour parvenir à ses fins $»^{50}$, ce qui ne contredit nullement son uniformité, car «[1]es Lois générales sont nécessaires, la Nature elle-même paraît s’y être soumise, mais elle peut employer des matières qui ne sont pas en notre disposition, et elle sait s'en servir d'une manière qu'il ne nous est tout au plus permis que de connaître $»^{51}$.

La machine universelle de Fontenelle est bien une natura naturans, une réalité en permanente transformation, et dont les infinis possibles sont comparables aux infinis mathématiques que Fontenelle défend ailleurs, et auxquels il a consacré près de trente années de recherche et un ouvrage, les Éléments de la géométrie de l'infini, parus en 1727. Il est vrai que les sciences mathématiques et les sciences physiques diffèrent par les efforts qu'elles demandent à l'esprit humain et par le discours qui en découle, mais elles ne participent pas moins toutes les deux de «l'esprit géométrique» qui semble présider à l'esthétique fontenellienne. De sorte que si la Nature sensible n'est pas toujours (ou pas encore) traduisible en langage mathématique, elle devient mathématique en action, une fonction exponentielle des possibilités de la matière et des formes sensibles qu'elle peut prendre.

L'esthétique du «faire voir » est donc la forme discursive qui répond à «l'esprit géométrique » qui se manifeste dans la Nature et que l'on peut définir dans des termes qui relèvent d'une logique mathématique. La Nature agit par des principes constants (des lois), mais dont les infinies opérations combinatoires produisent une infinité de structures possibles, les «merveilles » de la Nature, celles-là même qui constituent le «spectacle » par lequel commence toute la connaissance :

\footnotetext{
49 «Sur la structure des reins », HARS pour 1705, p. 46.

${ }^{50}$ «Sur les insectes », HARS pour 169, p. 39.

${ }^{51}$ «Sur la formation de la voix », HARS pour 1707, p. 20.
} 
Nous pouvons [...] avancer [...] qu'on ne saurait guère attribuer à la Nature trop d'uniformité dans les règles générales, et trop de diversité dans les applications particulières. Plus on étend son plan en y faisant entrer différentes combinaisons des mêmes principes, plus on est en droit de se croire dans la route de la vérité ${ }^{52}$.

Or, dans cette dynamique créatrice, le hasard devient même un des facteurs possibles de la diversification des formes. C'est d'ailleurs le hasard, cause aveugle par excellence, qui apparait, en dernier ressort, comme responsable de l'existence de certaines structures, dont les monstres $^{53}$, qui constituent l'une des thématiques les plus récurrentes de l'Histoire de l'académie des sciences :

Ce n'est que le hasard de la rencontre des fotus [...] qui les détermine à quitter certains chemins et à en suivre toujours d'autres. Et comme ce hasard est susceptible d'une infinité de combinaisons différentes, c'est une chose infinie que les monstres qui le sont par quelques parties doubles ${ }^{54}$.

\section{Ou encore :}

Ne reconnaît-on pas là [à l'origine des êtres exceptionnels que sont les monstres] les effets de causes accidentelles, irrégulières, aveugles, qui n'agissent pas de concert avec les lois générales et ne reviennent point deux fois à une même combinaison $?^{55}$

Il est vrai que la place du hasard dans le système fontenellien peut paraître problématique. Comme le remarque Jacques Roger, le hasard constitue une des explications possibles pour l'existence des monstres, sans que cela pose de problème d'ordre théologique, étant donné que la préexistence des germes, théorie de la génération la plus largement acceptée, suppose toujours l'intervention divine ${ }^{56}$. C'était d'ailleurs la thèse défendue par Malebranche, pour éviter le scandale que signifierait l'attribution de l'origine des monstres à la volonté de Dieu. Or, les intentions de Fontenelle ne semblent pas les mêmes : le hasard fontenellien n'a rien d'une excuse métaphysique, mais apparaît comme une loi possible de la nature, loi combinatoire «qui ne revien[t] point deux fois à une même combinaison », et teintée d'épicurisme. On pourrait certes également objecter qu'il s'agit là d'une aporie de la

\footnotetext{
52 «Observations botaniques », HARS pour 1702, p. 52.

${ }^{53}$ Maria Susana Seguin, «De la beauté des monstres : poétique de la catastrophe dans les sciences du vivant », dans Représenter la catastrophe à l'Âge classique : dispositifs, figures, motifs. Actes du colloque international tenu à Québec, 26-29 septembre 2007, Presses de l'Université Laval, à paraître.

54 «Observations d'anatomie », HARS pour 1702, p. 28.

55 «Sur les Monstres », HARS 1740, p. 49.

${ }^{56}$ Jacques Roger explique que Fontenelle s'écarte de la thèse malebranchiste, trop dépendante des impératifs finalistes, tout comme il rejette la thèse de Régis, qui attribue à Dieu la création des monstres, preuve de sa liberté et de sa puissance. La conclusion de Jacques Roger est que «Fontenelle [...] en cherche la cause [des monstres] dans les lois de la nature, dont tout hasard est exclu ». Or, comme on peut le voir, l'opinion de Fontenelle ne semble pas la même vers 1740, au moment où il écrit son dernier compte rendu sur la question. Jacques Roger, Les sciences de la vie dans la pensée française du XVIII siècle, op. cit., p. 397-418, et surtout p. 404.
} 
pensée fontenellienne, d'autant que les allusions au hasard apparaissent notamment dans les dernières années de son secrétariat à l'Académie des sciences. Mais quand on sait la constance avec laquelle Fontenelle préfère «suspendre son jugement » avant que de tomber dans une forme d'esprit de système ou de facilité intellectuelle, on est légitimement en droit de s'interroger sur les véritables intentions de l'auteur, qui laisse du moins entrevoir la possibilité d'une matière se contrôlant elle-même... ${ }^{57}$

Force est de constater que la grande machine universelle que l'auteur décrivait dans les Entretiens sur la pluralité des mondes prend dans les textes de l'Histoire de l'académie des sciences une dimension autrement polémique. L'auteur nous donne à voir une nature essentiellement matérielle, contrôlée par un ensemble de lois permettant, par le processus combinatoire, une infinité de possibles, dénués définitivement de toute connotation métaphysique ou transcendante. Ce principe combinatoire infini suppose aussi l'existence d'une matière dotée d'une énergie propre, dont Fontenelle ne définit jamais la nature dans l'Histoire de l'académie des sciences, mais qui semble capable de constamment se régénérer, de se transformer, renvoyant alors l'idée de la Création à un premier moment indéfini, éloignant toute idée d'une fin prochaine et, dans tous les cas, minimisant dangereusement la présence divine dans la scène naturelle.

Fontenelle réussit alors à établir une analogie parfaite entre les infinis mathématiques, l'infinitude de la nature et les possibilités infinies qui s'offrent à l'esprit humain dans sa dimension historique, tout en faisant de la règle combinatoire le principe épistémologique essentiel du savoir scientifique, du moins le moyen le plus sûr qui s'offre à l'esprit humain pour accéder progressivement à la connaissance positive du monde sensible, car « on se saurait observer les mêmes choses de trop d'endroits différents : à chaque nouveau point de vue la nature paraît nouvelle $»^{58}$. Sans cette référence, aucune connaissance ne semble possible. Certes, la nature sensible paraît insaisissable, et l'établissement d'un système général semble repoussé presque indéfiniment, comme l'affirmait la Préface sur l'utilité des mathématiques ${ }^{59}$ Mais dans cette logique infinitiste, ce «système général de la Nature ${ }^{60}$ ne paraît pas vraiment impossible ${ }^{61}$.

\footnotetext{
${ }^{57}$ Ce point mériterait des recherches complémentaires, notamment à propos de la relation de Fontenelle avec la pensée scientifique et philosophique des années 1730-1750, et son rapport à la génération des encyclopédistes et d'une nouvelle forme de matérialisme.

58 «Sur les réfractions », HARS pour 1700, p. 112.

59 «Jusqu'à présent l'Académie des Sciences ne prend la Nature que par petites parcelles. Nul Système général, de peur de tomber dans l'inconvénient des systèmes précipités, dont l'impatience de l'esprit humain ne s'accommode que trop bien [...] », HARS 1699, « Préface », p. XIX.
} 
Comme l'a montré Michel Blay, l'idée de l'infini constitue un pilier essentiel de la pensée fontenellienne ${ }^{62}$, mais elle acquiert une dimension encore plus radicale dans les volumes de l'Histoire de l'académie des sciences, car il n'y est pas question d'opposer indéfini et infini, comme le fait Descartes, tout comme il n'y a pas de distinction entre deux sortes d'infinis, l'un intellectuel, l'infini géométrique, l'autre transcendant, l'infini métaphysique, comme dans les Éléments de la géométrie de l'infini. La nature infinie de Fontenelle n'a aucune connotation transcendante, elle n'est, dans une logique mécaniste, que matière et mouvement. Contrairement à Pascal, l'univers infini n'effraie pas Fontenelle, il l'enchante ...

L'élaboration d'un savoir scientifique sur la nature se confond définitivement avec l'histoire de l'esprit humain, et l'écriture d'un discours du savoir avec l'écriture de cette histoire, celle-là même que Fontenelle est en train d'écrire pour l'Académie royale des sciences. La dynamique du savoir ainsi présentée ouvre des perspectives cognitives insoupçonnées, inscrites désormais dans une temporalité élargie, « un infini d'infini », comme disait Fontenelle ${ }^{63}$, et donc dégagée de toute menace apocalyptique. Fontenelle inscrit Nature et discours sur la Nature dans une dynamique en continuelle expansion. Et, par une dernière analogie, il fait de ce mouvement l'objet d'un plaisir esthétique mais aussi intellectuel, comparable à celui de l'Opéra observé par des «machinistes » : plaisir de la nature (ou de la matière ?), dotée ici d'un attribut purement divin, diversifiant éternellement ses formes, lançant perpétuellement de nouveaux défis à l'esprit de l'homme; plaisir du philosophe, tentant sans cesse d'appréhender les secrets de la nature, même s'il sait qu'elle lui échappera toujours un peu. Mais cette insatisfaction permanente que le savant cherche malgré tout à contenter, n'est-elle pas aussi une source ou un moteur du plaisir?

Maria Susana Seguin

Université Paul-Valéry Montpellier III

IRCL - UMR 5186 du CNRS

\footnotetext{
${ }^{60}$ «Sur les truffes », HARS pour 1711, p. 41.

${ }^{61}$ «Le temps viendra peut-être que l'on joindra en un corps régulier ces membres épars ; et s'ils sont tels qu'on le souhaite, ils s'assembleront en quelque sorte d'eux-mêmes. Plusieurs vérités séparées, dès qu'elles sont en assez grand nombre, offrent si vivement à l'esprit leurs rapports et leur mutuelle dépendance, qu'il semble qu'après avoir été détachées par une espèce de violence les unes d'avec les autres, elles cherchent naturellement à se réunir », HARS 1699, «Préface », p. XIX.

${ }^{62}$ Michel Blay, Les Raisons de l'infini. Du monde clos à l'univers infini. Paris, Gallimard, 1993 et plus récemment «Dire l'infini de Giordano Bruno à Fontenelle », Revue Fontenelle, $\mathrm{n}^{\circ} 4-2006$, Publications des Universités de Rouen et du Havre, 2006, p. 131-146.

${ }^{63}$ «Sur la fécondité des plantes », HARS pour 1701, p. 76.
} 